\title{
Comparison of the prevalence of islet autoantibodies according to age and disease duration in patients with type 1 diabetes mellitus
}

\author{
Young Hwa Kong, MD', \\ Min Sun Kim, MD, PhD ${ }^{1,2}$, \\ Dae-Yeol Lee, MD, PhD ${ }^{1,2}$
}

'Department of Pediatrics, Chonbuk National University Medical School, Jeonju, ${ }^{2}$ Research Institute of Clinical Medicine of Chonbuk National University-Biomedical Research Institute of Chonbuk National University Hospital, Jeonju, Korea

\begin{abstract}
Purpose: This study investigated the prevalence of islet autoantibodies in children and adults with T1DM according to their age and the duration of disease.

Methods: We measured the levels of islet autoantibodies, including antiglutamic acid decarboxylase antibody (anti-GAD Ab), and combined these with anthropometric measurements and laboratory tests of 137 patients newly diagnosed with T1DM during the last 20 years. The subjects were subdivided into four groups according to their age at the onset of the disease. We then compared the prevalence of islet autoantibodies in the different age groups with the duration of disease.

Results: Among the 137 patients, $68.9 \%$ tested positive for islet autoantibodies $(71.4 \%$ within 1 year; $67.7 \%$ after 1 year of the disease onset). Within 1 year of the onset of the disease, $66.3 \%$ of the patients were positive for the anti-GAD Ab, and $35.6 \%$ were positive for IAAs. The prevalence of islet autoantibodies was significantly higher in the prepubertal groups than in the postpubertal groups ( $80.0 \%$ vs. $58.3 \%)$. The rate of positive islet autoantibodies changed with the duration of disease, and it differed according to the type of autoantibody and the age of the patient.

Conclusion: The rates of positive islet autoantibodies were significantly higher in younger than in older patients at the time of the diagnosis of the disease. The positive rates were significantly changed 1 year after the onset of the disease in the preschool and the children groups. So these findings suggest that we need to diagnose type $1 \mathrm{~B}$ diabetes distinguished T2DM in aldolescent group, carefully.
\end{abstract}

Keywords: Islet autoantibody, Type 1 diabetes mellitus, Glutamic acid decarboxylase, Insulin autoantibody

\section{Introduction}

Type 1 diabetes mellitus (T1DM) is associated with insulin deficiency which is secreted from the pancreatic beta cells ${ }^{1)}$. T1DM is classified as either autoimmune (type IA) or idiopathic (type IB) diabetes. The major form of T1DM is type $1 \mathrm{~A}$, which occurs when autoantibodies attack and destroy pancreatic islet beta cells $(80-90 \%)$, causing the pancreas to produce little or no insulin. Islet autoantibodies are not present in type $1 \mathrm{~B}$ disease $\mathrm{e}^{2)}$.

Recently, the number of TIDM patients has increased in Korea and elsewhere ${ }^{3,4)}$. Although the incidence of T1DM in Korea is low compared to the rest of the world, the prevalence of the disease in children has increased in recent years ${ }^{3,5}$. According to reports, the incidence of T1DM in patients younger than 15 years in Seoul from 1985 to 1988 was 0.7 per 100,000/yr, and this has now increased to 1.35 per 100,000 per year ${ }^{6,7)}$. Some reports also showed that the age of onset of T1DM has decreased and that young patients with T1DM have a higher level of islet autoantibodies than older individuals ${ }^{8.9)}$.

In this study, we investigated the age of the patients at the onset of T1DM and the duration-
Received: 4 June, 2013

Revised: 24 June, 2013

Accepted: 25 June, 2013

Address for correspondence:

Dae-Yeol Lee, MD, PhD

Department of Pediatrics,

Chonbuk National University

Hospital, 634-18, Keumam-dong,

Jeonju, Jeonbuk 561-712, Korea

Tel: $+82-63-250-1469$

Fax: +82-63-250-1464

E-mail: leedy@jbnu.ac.kr
ISSN: 2287-1012(Print) ISSN: 2287-1292(Online)
This is an Open Access article distributed under the terms of the Creative Commons Attribution Non-Commercial License (http:// creativecommons.org/licenses/by-nc/3.0) which permits unrestricted non-commercial use, distribution, and reproduction in any medium, provided the original work is properly cited. 
specific incidence of islet autoantibodies in patients newly diagnosed with T1DM during the last 20 years in Jeollabuk-do.

\section{Materials and methods}

\section{Subjects}

The enrolled patients were newly diagnosed with T1DM in the Department of Pediatrics and Internal Medicine of Chonbuk National University Hospital between January 1991 and December 2010. The diagnostic criteria for T1DM included: 1) newly diagnosed T1DM with diabetic ketoacidosis, 2) a serum C-peptide level below $0.6 \mathrm{ng} / \mathrm{mL}$, and 3) if the serum C-peptide level was above $0.6 \mathrm{ng} / \mathrm{mL}$, the patient required insulin treatment to maintain blood glucose levels ${ }^{10)}$. We excluded the following: 1) when the type of diabetes changed to T2DM during the 6-month follow-up, 2) when the diabetes occurred secondary to other causes, 3) when the neonatal diabetes occurred, and 4) when there were no data on the concentrations of islet autoantibodies.

\section{Method}

We obtained demographic and laboratory data on the patients, such as age, gender, body mass index, serum glucose level, serum $\mathrm{pH}$, urine ketone, serum insulin, serum C-peptide, and glycosylated hemoglobin (HbAlc) level, from hospital records on the day they were newly diagnosed with T1DM. The patients' blood glucose levels in serum were measured at admission. Their insulin and C-peptide levels were analyzed in serum after fasting for 8 hours or more. The autoantibodies to glutamic acid decarboxylase (GAD), insulin, and islet cell autoantigen 512 (ICA512) were measured. The levels of autoantibodies were confirmed within 1 year of the onset of T1DM and 1 year after the diagnosis of the disease. When the patients were assessed the levels of autoantibodies more than 1 year after the diagnosis, we estimated the duration of T1DM. The concentrations of antiglutamic acid decarboxylase antibody (anti-GAD Ab) were measured by immunoradiometric assay using GAD Ab assay kit (RSR Ltd., Cardiff, UK), and patients were considered anti-GAD $\mathrm{Ab}$ positive when the concentration was above $0.9 \mathrm{unit} / \mathrm{mL}^{11)}$. Concentrations of insulin autoantibodies (IAAs) and ICA512 were measured by radioimmunoassay (RIA) using AIA-100 kit (Biosource, Nivelles, Belgium) and IA-2 Ab Cosmic kit (RSR Ltd.). An IAA level above 7\% was considered positive ${ }^{11}$. Patients were deemed positive for ICA512 at a level of 0.4 unit $/ \mathrm{mL}$.

To compare the concentration and the incidence of the islet autoantibodies, we classified the patients according to their ages. They were divided as follows: a preschool group (group I, 0-5 years old), an children group (group II, 6-12 years old), an adolescent group (group III, 13-19 years), and an adult group (group IV, above 20 years). We further subdivided them into two groups: prepuberty ( $0-12$ years) and postpuberty (older than 13 years).

\section{Statistical methods}

All data were analyzed using IBM SPSS ver. 18.0 (IBM Co., Armonk, NY, USA). The Student $t$ test, chi-square test, and one-way analysis of variance were used for the analysis of the variables. The Pearson correlation analysis assessed the relationship between the concentrations of the islet autoantibodies and the other parameters. The independent twosample $t$ test was used to compare the levels of autoantibodies within 1 year of the disease onset and 1 year after the diagnosis. All the variables are expressed as the mean \pm standard deviation (SD). For all analyses, $P<0.05$ was considered statistically significant.

\section{Results}

\section{Gender and age distribution}

One hundred thirty-seven patients with new-onset T1DM were enrolled. The study group consisted of 72 women (52.6\%) and 65 men $(47.5 \%)$. The mean age of the whole group was $16.17 \pm 9.89$ years old. There were 16 patients $(11.7 \%)$ in the preschool group (group I), 51 (37.2\%) in the children group (group II), 30 (21.9\%) in the adolescent group (group III), and 40 (29.2\%) in the adult group (group IV) (Table 1). The mean of disease duration was 5.41 years in patients measured 1 year after the diagnosis.

\section{Comparison of clinical and laboratory characteristics among each age group at diagnosis}

The serum glucose level was significantly different among the four groups $(P<0.05)$. It was particularly high in group I compared to the other groups $(499.9 \pm 286.1 \mathrm{mg} / \mathrm{dL})$. There was no significant difference among the groups with respect to gender and the incidence of clinical symptoms (polyuria, polydipsia, change of consciousness) at the time of diagnosis (data not shown). There was also no significant difference according to $\mathrm{HbAlc} \mathrm{level,} \mathrm{arterial} \mathrm{pH}$, and bicarbonate levels. In addition, there was no significant difference among the groups in terms of serum C-peptide and $\mathrm{HbAlc}$ levels. However, group I showed the lowest values for these parameters among the four groups (Table 1).

\section{Comparison of the incidence of positive islet autoantibodies between the prepuberty and postpuberty groups within 1 year of the diagnosis}

Islet autoantibodies were measured within four weeks of the diagnosis in most of patients, excepting just one case which was measured within eight weeks of the diagnosis. The overall prevalence of positive islet autoantibodies (anti-GAD $\mathrm{Ab}$, ICA512, or IAA) was $71.43 \%$. Of these, anti-GAD Ab was 
detected in $66.28 \%(57 / 86)$ of the patients, ICA512 was detected in $54.17 \%(13 / 24)$, and IAAs were present in $35.63 \%(31 / 87)$. There was a significant difference between the prepuberty and postpuberty groups $(P<0.05)$ in the prevalence of positive islet autoantibodies. The prevalence of islet autoantibody-positive patients in the prepuberty group $(80.00 \%)$ was significantly greater than in the postpuberty group (58.33\%). In addition, a significantly higher percentage of patients in the prepuberty group $(74.51 \%, 47.17 \%$, and $66.67 \%)$ tested positive for antiGAD Ab, ICA512, and IAA than in the postpuberty group (54.29\%, 17.65\%, and $16.67 \%$ ) (Table 2).

In the analysis of the mean levels of islet autoantibodies, no significant difference was observed among the groups with respect to the mean anti-GAD Ab and ICA512 concentrations, However, the mean IAA level was significantly greater in the prepuberty groups (group I and II) than in the postpuberty groups (group III and IV) $(P<0.001)$ (Fig. 1).

\section{Comparison of the prevalence of positive islet autoantibodies by disease duration}

The number of patients who tested positive for islet autoantibodies (anti-GAD Ab, ICA512, or IAA) within 1 year of the disease onset $(71.43 \%)$ and 1 year after $(67.68 \%)$ the diagnosis showed no significant difference (Table 3 ). There was no significant difference in the prevalence of ICA512-positive patients (data not shown, $P>0.05$ ). However, the prevalence of anti-GAD Ab-positive patients was significantly greater within one year of the onset of the disease than 1 year after the diagnosis. The prevalence of IAA-positive patients was significantly greater within one after the onset of the inset of the disease than 1 year after the diagnosis (Table 3, Fig. 3) $(P<0.05)$. Group I showed the highest percentage decrease (from $81.82 \%$ to $11.11 \%$ ) in the prevalence of anti-GAD Ab-positive autoantibodies, followed by group II (from $72.50 \%$ to $48.15 \%)(P<0.05)$. There was no significant difference in this parameter between group III and IV (Fig. 2) $(P>0.05)$. The overall prevalence of IAA-positive

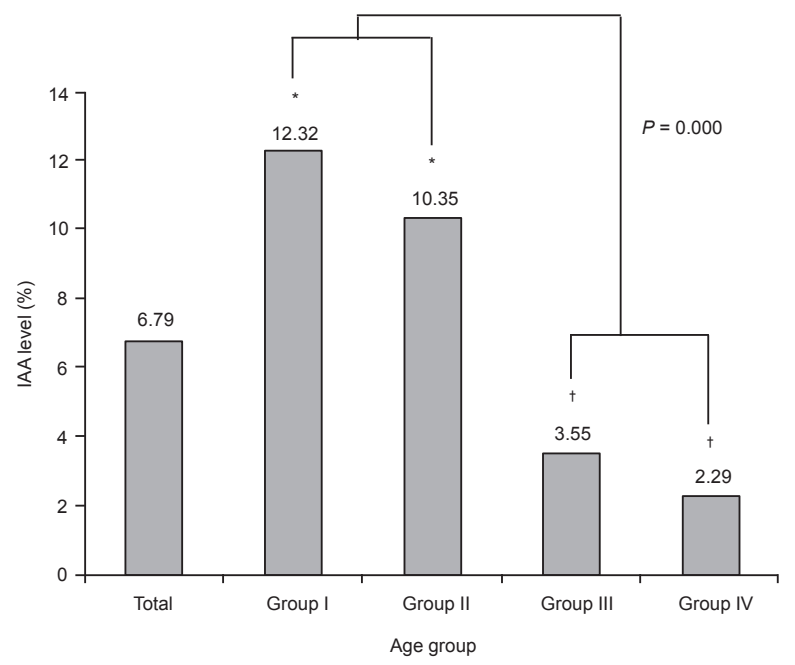

Fig. 1. Comparison of mean insulin autoantibody (IAA) mean levels among the each age groups in patients with type 1 diabetes diagnosed within 1 year of onset. " ${ }^{*}$ Statistical significances were tested by one way analysis of variances among groups. The same markers indicate nonsignificant difference between groups based on Duncan multiple comparison test.

Table 1. Characteristics among the each age groups in patients with type 1 diabetes

\begin{tabular}{lcccccc}
\hline Characteristic & Total & Group I & Group II & Group III & Group IV & P-value \\
\hline Age (yr) & $16.17 \pm 9.89(137)$ & $3.24 \pm 1.44(16)$ & $10.07 \pm 1.86(51)$ & $16.41 \pm 2.33(30)$ & $28.92 \pm 6.90(40)$ & 0.000 \\
BMI (SDS) & $-0.79 \pm 1.72(132)$ & $-1.21 \pm 2.87(15)$ & $-0.59 \pm 1.59(51)$ & $-0.59 \pm 1.33(30)$ & $-1.08 \pm 1.57(36)$ & 0.391 \\
HbA1c (\%) & $11.95 \pm 2.97(131)$ & $10.63 \pm 2.72(16)$ & $12.28 \pm 2.26(50)$ & $12.04 \pm 3.01(28)$ & $11.99 \pm 3.75(37)$ & 0.281 \\
C-peptide $(\mathrm{ng} / \mathrm{mL})$ & $0.94 \pm 0.92(132)$ & $0.40 \pm 0.35(16)$ & $1.00 \pm 0.62(50)$ & $0.98 \pm 0.77(29)$ & $1.06 \pm 1.38(37)$ & 0.095 \\
Glucose $(\mathrm{mg} / \mathrm{dL})$ & $402.85 \pm 229.72(132)$ & $499.87 \pm 286.13(15)^{*}$ & $431.98 \pm 189.08(51)^{\dagger}$ & $422.00 \pm 290.04(29)^{\dagger}$ & $308.34 \pm 174.74(37)^{\ddagger}$ & 0.017 \\
Arterial pH & $7.30 \pm 0.15(104)$ & $7.33 \pm 0.12(13)$ & $7.30 \pm 0.15(44)$ & $7.29 \pm 0.17(19)$ & $7.30 \pm 0.13(28)$ & 0.914 \\
$\mathrm{HCO}_{3}(\mathrm{mmol} / \mathrm{L})$ & $16.59 \pm 9.31(95)$ & $15.9 \pm 6.68(13)$ & $16.00 \pm 9.48(44)$ & $17.61 \pm 9.98(19)$ & $17.39 \pm 10.29(19)$ & 0.897 \\
\hline
\end{tabular}

Values are presented as mean \pm standard deviation (no. of patients).

$\mathrm{BMI}$, body mass index; HbA1c, glycosylated hemoglobin; $\mathrm{HCO}$, hydrogen carbonate.

${ }^{*},+$, Statistical significances were tested by one way analysis of variances among groups. The same markers indicate nonsignificant difference among groups based on Duncan multiple comparison test.

Table 2. Prevalence of pancreatic autoantibodies between prepuberty and postpuberty group in patients with type 1 diabetes diagnosed within 1 year of onset

\begin{tabular}{|c|c|c|c|c|}
\hline & \multicolumn{3}{|c|}{ Prevalence } & \multirow{2}{*}{$P$-value } \\
\hline & Total & Prepuberty & Postpuberty & \\
\hline Anti-GAD Ab & $66.28(57 / 86)$ & $74.51(38 / 51)$ & $54.29(19 / 35)$ & 0.042 \\
\hline IAA & $35.63(31 / 87)$ & $47.17(25 / 53)$ & $17.65(6 / 34)$ & 0.003 \\
\hline ICA512 & $54.17(13 / 24)$ & $66.67(12 / 18)$ & $16.67(1 / 6)$ & 0.033 \\
\hline Anti-GAD Ab or IAA or ICA512 & $71.43(65 / 91)$ & $80.00(44 / 55)$ & $58.33(21 / 36)$ & 0.025 \\
\hline
\end{tabular}

Values are presented as prevalence rate (no. of patients).

Anti-GAD Ab, antiglutamic acid decarboxylase antibody; IAA, insulin autoantibody; ICA512, islet cell autoantigen 512. 
autoantibodies increased significantly from $35.63 \%$ within 1 year of the diagnosis to $53.41 \% 1$ year after the diagnosis. The highest increase was observed in group I (41.67\% to $92.31 \%$ from) and group II (48.78\% to $72.22 \%)$. Group III and IV showed no significant difference in the overall prevalence of IAA-positive autoantibodies (Fig. 3) .

\section{Correlation of levels of islet autoantibodies with laboratory findings}

The levels of HbAlc at the time of measurement of islet autoantibodies were not correlated with anti-GAD Ab levels and IAA levels. Also, there was no significant correlation between the levels of islet autoantibodies and any of the other laboratory findings (data not shown).

\section{Discussion}

DM has been steadily increasing in children and adolescents worldwide. Accordingly, there is a need for early diagnosis and appropriate treatment or management. There has been some research on methods to diagnose the disease while it is still at an early stage ${ }^{12-14)}$. As described earlier, T1DM is separated type $1 \mathrm{~A}$ diabetes for immune mediated diabetes and type $1 \mathrm{~B}$ diabetes for nonimmune diabetes with severe insulin deficiency ${ }^{1,2)}$. And T2DM is idiopathic diabetes with insulin resistance. The studies have shown that the majority of DM in children and adolescents is $\mathrm{T} 1 \mathrm{DM}^{1)}$. But the recent rise in the numbers of cases of T2DM highlights the need to distinguish T1DM and T2DM ${ }^{15)}$. Besides the age of patients at the onset of the disease and their clinical symptoms, it can be difficult to distinguish T1DM and T2DM. So, the following has been used to classify the type of DM: insulin, C-peptide, and ketoacidosis in serum and ketone

Table 3. Prevalence rate of pancreatic autoantibodies in patients with type 1 diabetes between diagnosed within 1 year and after 1 year

\begin{tabular}{|c|c|c|c|c|c|c|}
\hline & \multicolumn{5}{|c|}{ Prevalence } & \multirow{2}{*}{$P$-value } \\
\hline & Total & Group I & Group II & Group III & Group IV & \\
\hline \multicolumn{7}{|c|}{ Anti-GAD Ab } \\
\hline$<1$ year & $66.28(57 / 86)$ & $81.82(9 / 11)$ & $72.50(29 / 40)$ & $53.33(8 / 15)$ & $55.00(11 / 20)$ & 0.254 \\
\hline$\geq 1$ year & $39.24(31 / 79)$ & $11.11(1 / 9)$ & $48.15(13 / 27)$ & $31.82(7 / 22)$ & $47.62(10 / 21)$ & 0.308 \\
\hline \multicolumn{7}{|l|}{ IAA } \\
\hline$<1$ year & $35.63(31 / 87)$ & $41.67(5 / 12)^{*}$ & $48.78(20 / 41)^{*}$ & $26.67(4 / 15)^{\dagger}$ & $10.53(2 / 19)^{\ddagger}$ & 0.027 \\
\hline$\geq 1$ year & $53.41(47 / 88)$ & $92.31(12 / 13)^{*}$ & $72.22(26 / 36)^{*}$ & $35.00(7 / 20)^{\dagger}$ & $10.53(2 / 19)^{\dagger}$ & 0.000 \\
\hline \multicolumn{7}{|c|}{ Anti-GAD Ab or IAA or ICA512 } \\
\hline$<1$ year & $71.43(65 / 91)$ & $84.62(11 / 13)$ & $78.57(33 / 42)$ & $66.67(10 / 15)$ & $52.38(11 / 21)$ & 0.110 \\
\hline$\geq 1$ year & $67.68(67 / 99)$ & $85.71(12 / 14)^{*}$ & $77.50(31 / 40)^{\dagger}$ & $54.17(13 / 24)^{\ddagger}$ & $52.38(11 / 21)^{\ddagger}$ & 0.043 \\
\hline
\end{tabular}

Values are presented as prevalence rate (no. of patients).

Anti-GAD Ab, antiglutamic acid decarboxylase antibody; IAA, insulin autoantibody; ICA512, islet cell autoantigen 512.

${ }^{*},+$, Statistical significances were tested by one way analysis of variances among groups. The same markers indicate nonsignificant difference among groups based on Duncan multiple comparison test.

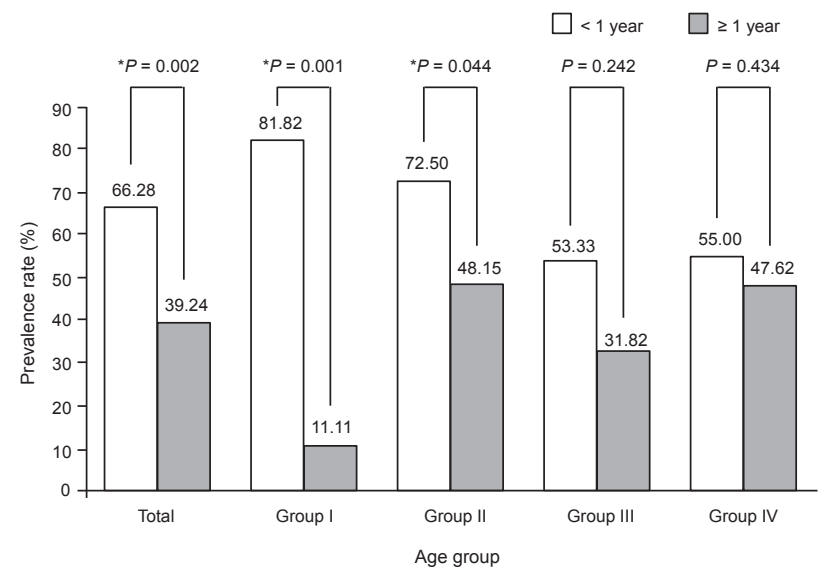

Fig. 2. Prevalence rate (\%) of antiglutamic acid decarboxylase autoantibody in patients with type 1 diabetes diagnosed within 1 year and after 1 year of onset. *The marker indicates statistical significant difference between groups diagnosed within 1 year and after 1 year of onset. Statistical significances were tested by independent two-sample $t$ test between groups.

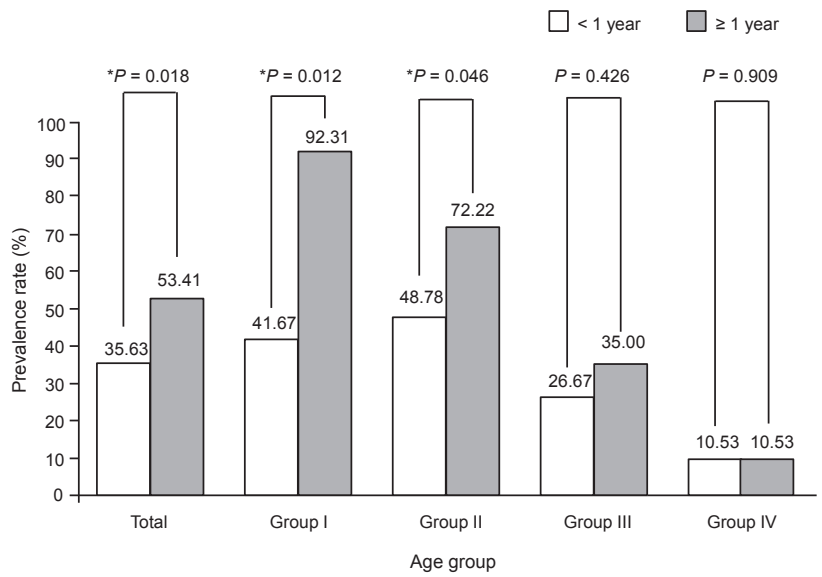

Fig. 3. Prevalence rate (\%) of insulin autoantibody (IAA). The first group patients studied IAA within one year after diagnosis of type 1 diabetes. And the second group patients studied after one year. *The marker indicates statistical significant difference between groups diagnosed within 1 year and after 1 year. Statistical significances were tested by independent two-sample $t$ test between groups. 
bodies in urine ${ }^{16)}$. Several studies ${ }^{17-20)}$ reported the presence of ketoacidosis in patients with T2DM; they also reported that the age at which patients develop T2DM is decreasing, with younger patients now presenting with this disease. These findings contribute to the difficulty in differentiating between the two types of DM and to the change in the diagnosis during the follow-up. Recently, the test on autoantibody-related with pancreas has been developed for the purpose of the classification, diagnosis, and treatment of $\mathrm{DM}^{12-14)}$.

Lee et al. ${ }^{12)}$ reported that 50-70\% of T1DM patients tested positive for islet autoantibodies at the time of diagnosis and that the rate decreased as DM progressed. In our study, 68.9\% tested positive for islet autoantibodies within 1 year of being diagnosed, and the rate slightly decreased 1 year later. The incidence of positive islet autoantibodies differed, depending on the age group. Within 1 year of the diagnosis, the positive rate of autoantibodies decreased according to the age group: $84.6 \%$ in the preschool group, $78.6 \%$ in the children group, $66.7 \%$ in the adolescent group, and $52.4 \%$ in adult group. In the comparison between the prepuberty and the postpuberty groups, the prevalence of anti-GAD Ab-, ICA512-, and IAA-positive patients was higher in the prepuberty groups than in the postpuberty groups. Previous studies ${ }^{21,22)}$ reported that younger subjects showed higher levels of islet autoantibodies to T1DM. However, the cause was not clearly identified ${ }^{21,22)}$. The research by Kordonouri et al. ${ }^{21)}$ showed that concentrations of anti-GAD $\mathrm{Ab}$ and IA-2 Ab were higher in a pubertal group than in an adult group, both of which were healthy and not diagnosed with DM. This difference in the positive rate of islet autoantibodies among the age groups is also seen in diseases other than T1DM, with one study reporting apparent differences in the concentration of total immunoglobulin among different age groups, suggesting that antibody concentrations can vary among different age groups $^{23)}$. However, further analyses are needed to confirm this.

The ICA, which was the first pancreatic autoantibody to be found, shows a peak concentration during the early stages of T1DM and slowly decreases afterwards ${ }^{24,25)}$. The concentration of anti-GAD Ab also decreases as T1DM progresses. However, some reports ${ }^{24)}$ indicate that the anti-GAD Ab shows a slower reduction in titer than ICA. Therefore, it can be detected for an extended period after the onset of T1DM. However, in the present study, the positive rate of pancreatic autoantibodies showed a sharp decrease from $66.3 \%$ to $39.2 \%$ within and after 1 year of the diagnosis, respectively. This decrease in the positive rate of autoantibodies was particularly prominent in the preschool group and not as noticeable in the adult group. This result is thought to be related to the destruction of pancreatic beta cells. In other words, as the disease progresses and the number of pancreatic beta cells decrease, the autoantibody titer shows a sharp decrease, as there is no more antigen to respond ${ }^{26)}$.

In patients with T1DM, anti-insulin antibody is produced through two different mechanisms. In the first mechanism, an autoimmune response leads to the production of IAA. In the second mechanism, the immune response to external sources of insulin results in the production of insulin antibody.
Some studies reported ${ }^{27)}$ that antibody production following insulin injections was not a significant factor in the regulation of diabetes. However, others ${ }^{28)}$ suggested that anti-insulin antibodies can considerably affect the biological activity of insulin. Therefore, the clinical importance of anti-insulin antibodies remains controversial. IAA is produced in an antigenic manner during the destruction of pancreatic beta cells and IAA-positive patients become IAA negative shortly after the onset of the disease. IAA is thought to cause cross-reactions with anti-insulin antibodies. The RIA used to measure IAA in this study cannot differentiate IAA from the insulin antibodies formed after insulin injections ${ }^{29,30)}$. According to our study, the positive rate of IAA was $35.6 \%$ in the follow-up period less than 1 year after the diagnosis of the disease, and it increased up to $53.4 \%$ after more than 1 year. However, most of the patients who were IAA-positive within 1 year of the diagnosis were diagnosed when the disease was at an early stage and before they had received insulin treatment. Thus, the prevalence of positive IAA was actually $35.6 \%$, and the prevalence of IAA after more than 1 year after diagnosis of T1DM, including the antiinsulin antibody, was $53.4 \%$. Consequently, the incidence of IAA-positive patients at the time of diagnosis was significantly higher in the prepuberty groups than in the postpuberty groups, and the incidence was increased significantly in the preschool and children groups more than 1 year after the diagnosis.

In our study, $\mathrm{HbAlc}$ was not associated with anti-GAD Ab and IAA, as another study found no significant relationship between the concentrations of islet autoantibodies and other clinical charicteristics ${ }^{29)}$.

The positive rates of islet autoantibodies differed, depending on the duration of diabetes and the age of the patient at the onset of the disease. However, additional study is needed to confirm this finding due to the following limitations of the our study: the follow-up period was too short, the size of the group was too small, and the method of IAA interpretation cannot differentiate IAA from the insulin antibodies formed after insulin injections.

Consequently, the positive rate of islet autoantibodies in T1DM was significantly lower in the adolescent and adult groups than the preschool and children groups. So carefully, we need to diagnose type $1 \mathrm{~B}$ diabetes distinguished T2DM in aldolescent group. Based on our findings, islet autoantibodies may aid the differential diagnosis of diabetes at an early stage of the disease. In addition, the positive rate of anti-GAD Ab and IAA were significantly changed in accordance with the increase in the duration of the disease. So, additional study is needed to shed light on the relationship between the etiology of T1DM and autoantibodies. Future studies of autoantibody tests, such as the anti-GAD Ab test, should also be conducted to diagnose high-risk groups of diabetes patients without symptoms.

\section{Conflict of interest}

No potential conflict of interest relevant to this article was reported. 


\section{Acknowledgments}

This work was supported by the Chonbuk National University Hospital research fund 2010.

\section{References}

1. Expert Committee on the Diagnosis and Classification of Diabetes Mellitus. Report of the expert committee on the diagnosis and classification of diabetes mellitus. Diabetes Care 2003;26 Suppl 1:S5-20.

2. Yoo EG, Shin HJ, Kim DH. The clinical types and characteristics of diabetes mellitus in Korean children. J Korean Pediatr Soc 2000;43:1591-8.

3. DIAMOND Project Group. Incidence and trends of childhood type 1 diabetes worldwide 1990-1999. Diabet Med 2006;23:857-66.

4. Patterson CC, Dahlquist GG, Gyurus E, Green A, Soltesz G; EURODIAB Study Group. Incidence trends for childhood type 1 diabetes in Europe during 1989-2003 and predicted new cases 2005-20: a multicentre prospective registration study. Lancet 2009;373:2027-33.

5. Shin $\mathrm{CH}$. Epidemiologic characteristics of type 1 diabetes in children aged 14 years or under in Korea, 1985-2000. Korean J Pediatr 2008;51:569-75.

6. Ko KW, Yang SW, Cho NH. The incidence of IDDM in Seoul from 1985 to 1988. Diabetes Care 1994;17:1473-5.

7. Shin CH. Type 1 diabetes mellitus. J Korean Pediatr Soc 2002;45:1181-91.

8. Kim MS, Yu KY, Na JI, Kim JD, Lee OK, Lee DY. The changes of incidence of childhood diabetes in Jeollabuk-do for 26 years. J Korean Soc Pediatr Endocrinol 2008;13:2935.

9. Sella T, Shoshan A, Goren I, Shalev V, Blumenfeld O, Laron $\mathrm{Z}$, et al. A retrospective study of the incidence of diagnosed Type 1 diabetes among children and adolescents in a large health organization in Israel, 2000-2008. Diabet Med 2011;28:48-53.

10. Hur J, Lee HS, Hwang JS. Clinical characteristics of type 1 diabetes mellitus at initial diagnosis. J Korean Soc Pediatr Endocrinol 2006;11:177-84.

11. Choi JH, Kim MS, Kim CJ, Kim JD, Lee DY. Comparison of clinical and laboratory characteristics in children with type 1 diabetes according to pancreatic autoantibodies. Korean J Pediatr 2010;53:414-9.

12. Lee CW, Shin HJ, Kim DH. Prevalence of autoimmune antibodies in type i diabetic children and their siblings. J Korean Soc Pediatr Endocrinol 1999;4:78-87.

13. Ludvigsson J, Faresjo M, Hjorth M, Axelsson S, Cheramy $\mathrm{M}$, Pihl M, et al. GAD treatment and insulin secretion in recent-onset type 1 diabetes. N Engl J Med 2008;359:190920.

14. Hong EH, Park JS, Lee HS, Cho MH, Ko CW. Clinical characteristics and laboratory findings of children who were newly diagnosed with diabetes mellitus (from 2001 to
2008). J Korean Soc Pediatr Endocrinol 2009;14:110-5.

15. D'Adamo E, Caprio S. Type 2 diabetes in youth: epidemiology and pathophysiology. Diabetes Care 2011;34(Suppl 2):161S-165S.

16. Park J, Oh J, Yu J. Autoantibody positivity and clinical characteristics of diabetes mellitus in childhood. J Korean Soc Pediatr Endocrinol 2011;16:119-27.

17. Yu J, Jin H, Ko J, Kang H. A case of severe diabetic ketoacidosis in a child with type 2 diabetes. J Korean Soc Pediatr Endocrinol 2011;16:46-50.

18. Seo JY, Bae SH, Woo YJ, Kim CJ. The precipitating factor and clinical features of diabetic ketoacidosis. Chonnam Med J 2010;46:94-8.

19. Kang S, Kim S, Lee J. Prevalence rates and risk factors of overweight and obesity in children and adolescents: using Korean National Health and Nutrition Examination Survey 2005 data. Korean J Epidemiol 2008;30:188-97.

20. Choi J, Ko JM, Lee JH, Choi JH, Yoo HW. Clinical characteristics of type 2 diabetes in children and adolescents. J Korean Soc Pediatr Endocrinol 2006;11:64-9.

21. Kordonouri O, Hartmann R, Gruters-Kieslich A, Knip M, Danne T. Age-specific levels of diabetes-related GAD and IA-2 antibodies in healthy children and adults. J Pediatr Endocrinol Metab 2002;15:47-52.

22. Betterle C, Lazzarotto F, Fusari A, Zanchetta R, Benedini S, Pedini B, et al. Pancreatic autoantibodies in Italian patients with newly diagnosed type 1 diabetes mellitus over the age of 20 years. Acta Diabetol 2006;43:79-83.

23. Buckley CE 3rd, Dorsey FC. Serum immunoglobulin levels throughout the life-span of healthy man. Ann Intern Med 1971;75:673-82.

24. Lee SH, Yoon JS, Eun MJ, Kim JH, Park YH, Won KC, et al. Five year follow-up of ICA and GADA in childhood onset type 1 DM. J Korean Diabetes Assoc 2003;27:395-404.

25. Park YS. Prospective follow-up of autoantibody prevalences in patients with type 1 diabetes. J Korean Diabetes Assoc 2003;27:391-4.

26. Decochez K, Tits J, Coolens JL, Van Gaal L, Krzentowski G, Winnock F, et al. High frequency of persisting or increasing islet-specific autoantibody levels after diagnosis of type 1 diabetes presenting before 40 years of age. The Belgian Diabetes Registry. Diabetes Care 2000;23:838-44.

27. Asplin CM, Hartog M, Goldie DJ, Alberti KG, Smythe P, Binder $\mathrm{C}$, et al. A comparison between diabetics receiving a high or low daily insulin dosage. Horm Metab Res 1978;10:365-9.

28. Francis AJ, Hanning I, Alberti KG. The influence of insulin antibody levels on the plasma profiles and action of subcutaneously injected human and bovine short acting insulins. Diabetologia 1985;28:330-4.

29. Lee K, Lee SY, Song CK, Jung MH, Yi KH, Hong JY, et al. Factors associated with the development of anti-insulin antibody in diabetic children. J Korean Soc Pediatr Endocrinol 2000;5:100-6.

30. Kurtz AB, Nabarro JD. Circulating insulin-binding antibodies. Diabetologia 1980;19:329-34. 